\title{
Optimum Dose of Spinal Ropivacaine with or Without Single Intravenous Bolus of S-Ketamine During Elective Cesarean Delivery: A Randomized, Sequential Dose-Finding Study
}

\author{
Xiaoyu Zhang \\ the International Peace Maternity and Child Health Hospital, Shanghai Jiao Tong University \\ Jianwei Wang \\ the International Peace Maternity and Child Health Hospital, Shanghai Jiao Tong University \\ Xiao-Hu An \\ the International Peace Maternity and Child Health Hospital, Shanghai Jiao Tong University \\ Yu-Chieh Chao \\ the International Peace Maternity and Child Health Hospital, Shanghai Jiao Tong University

\section{Yong Bian} \\ Shanghai Children's Medical Center

\section{Zifeng Xu} \\ the International Peace Maternity and Child Health Hospital, Shanghai Jiao Tong University \\ Tao Xu ( $\nabla$ towerxutao@163.com ) \\ the International Peace Maternity and Child Health Hospital, Shanghai Jiao Tong University
}

\section{Research Article}

Keywords: ED90, ropivacaine, post-spinal hypotension, S-ketamine, cesarean delivery, post-operative analgesia

Posted Date: August 9th, 2021

DOI: https://doi.org/10.21203/rs.3.rs-737523/v1

License: (c) (i) This work is licensed under a Creative Commons Attribution 4.0 International License. Read Full License 


\section{Abstract \\ Background}

Post-spinal hypotension has a high occurrence during cesarean delivery, and can lead to adverse maternal or fetal outcomes. The purpose of the study was to determine the optimal dose of spinal ropivacaine for cesarean section with or without intravenous single bolus of S-ketamine and to observe the incidences of hypotension of both methods.

\section{Methods}

Eighty women undergoing elective cesarean delivery were randomly allocated into Group ropivacaine or Group ropivacaine with intravenous S-ketamine. If the upper sensory level of the patient reached T6 and the visual analogue scale (VAS) scores continuously below 3 before delivery, the next patient had a 1/9th chance of receiving a lower dose or an 8/9th chance of receiving the same of previous dose. If the patient had VAS scores above 2 or needed an extra epidural rescue bolus before delivery, a higher dose was used for the next patient. The primary outcome was the successful use of spinal ropivacaine to maintain VAS of patients below 3 before delivery and the incidence of post-spinal hypotension of both groups.

Secondary outcomes included the incidences of hypotension related symptoms and managements, upper sensory level of anesthesia, level of sedation, neonatal outcomes, Edinburgh postnatal depression scale scores on admission and discharging of hospital and post-operative analgesic effect. The $90 \%$ effective dose (ED90) and $95 \%$ confidence interval $(95 \% \mathrm{Cl})$ were estimated by isotonic regression.

\section{Results}

The estimated ED90 of ropivacaine was $11.8 \mathrm{mg}(95 \% \mathrm{Cl} 11.7-12.7)$ with and $14.7 \mathrm{mg}(95 \% \mathrm{Cl} 14.6-$ 16.0) without intravenous S-ketamine using biased coin up-down sequential dose-finding method, and the incidences of hypotension and its related symptoms were significantly lower in S-ketamine group.

\section{Conclusions}

A spinal dose of ropivacaine $12 \mathrm{mg}$ with single intravenous $0.15 \mathrm{mg} / \mathrm{kg}$ bolus dose of S-ketamine may significantly reduce the risk of hypotension and provide sedative status before delivery, thus the method could be cautiously used in parturients with high risks of hypotension or extreme nervousness.

\section{Clinical trial registration:}

http://www.chictr.org.cn (ChiCTR2000040375; 28 Nov 2020) 


\section{Background}

Maternal hypotension is the most common side effect of spinal anesthesia during cesarean delivery, accounting for $70-83 \%$ of all cases. ${ }^{1-3}$ It may increase the risk of adverse maternal and foetal outcomes, such as maternal nausea, vomiting, dyspnoea, low Apgar scores, and foetal acidosis. ${ }^{4-7}$ Thus, hypotension prevention is paramount to good outcomes. Methods such as intrathecal adjuvant opioid use to reduce the total dose of anaesthetics delivered, ${ }^{8}$ prophylactic use of vasopressors, ${ }^{4,9}$ and the use of intravenous (IV) pre-load colloid liquid ${ }^{10}$ have been proposed to reduce the incidence or extent of maternal hypotension after spinal anesthesia during cesarean delivery.

S-ketamine is an $\mathrm{S}+$ isomer of ketamine that is twice as potent as ketamine ${ }^{11}$; it achieves sedation, analgesia, and sympathetic activation ${ }^{12}$ with a relatively low risk of side effects to mothers or infants, ${ }^{13}$ making it an optimum choice as an IV adjuvant for spinal anesthesia during cesarean delivery. To reduce the risk of psychomimetic reactions, S-ketamine administration was limited to an ultra-low single dose in the present study.

We hypothesized that a single ultra-low IV bolus of S-ketamine may reduce the total need for spinal ropivacaine and thus reduce the incidence of post-spinal anesthesia hypotension during cesarean delivery. Finally, $90 \%$ effective doses (ED90) of spinal ropivacaine with and without a single IV bolus of Sketamine were estimated, using the biased coin up-down (BCUD) sequential dose-finding method; the incidence of post-spinal anesthesia hypotension was observed.

\section{Methods}

This was a prospective, double-blind, randomised, BCUD-based, sequential dose-finding study was approved by China Ethics Committee of Registering Clinical Trials, West Hospital, Sichuan University, Sichuan, China on 29 September 2020 (Chairperson Taixiang Wu, Ethical No.: ChiECRCT20200301) and registered at http://www.chictr.org.cn (ChiCTR2000040375; 28 Nov 2020). A total of 85 women, aged 2240 years, and with a full-term ( $>37$ weeks of gestation) singleton pregnancy, the American Society of Anaesthesiologists (ASA) physical status II, and undergoing an elective cesarean delivery were recruited during Jan 2021 and April 2021 at the International Peace Maternity and Child Health Hospital. After providing signed informed consent, the women were allocated by a research assistant to one of two groups, using a random number generator in SPSS for Windows version 18.0 (SPSS Inc., Chicago, IL, USA), specifically, the ropivacaine (R) and ropivacaine with intravenous S-ketamine (RS) groups. All patients were blinded to group allocation.

Exclusion criteria were as follows: contraindications to combined spinal and epidural (CSE) anesthesia; the ASA score of III-IV; allergy to ketamine, S-ketamine, or ropivacaine; history of gastroesophageal reflux, full stomach, placenta previa, placental abruption, pre-existing or pregnancy-induced hypertension, preeclampsia, cardiovascular or cerebrovascular disease; foetal distress or abnormalities; multiple gestations; refusal to participate, and emergent cesarean delivery. Due to differences in delivery time and 
uterine contraction pain levels after surgery, women who had previously undergone a cesarean section and multiparas were excluded.

Patients were recruited for the study on the day before surgery in the obstetrical wards, when they were asked to complete the Edinburgh Postnatal Depression Scale (EPDS), which is a useful screening instrument for detection of women at risk for depression in the perinatal period. ${ }^{14}$ All patients were instructed to fast from midnight on the day of surgery. After entering the operating room, an IV line was established with an 18G IV catheter in the left forearm, and lactated Ringer's solution was infused at a rate of $1 \mathrm{ml} \cdot \mathrm{min}^{-1}$ to maintain the vein open.

Routine monitoring was performed continuously, including electrocardiography, non-invasive blood pressure, and pulse oximetry. Systolic blood pressure (SBP), heart rate (HR), and pulse oximetry were assessed every $2 \mathrm{~min}$. The first two resting SBP and HR measurements obtained in the supine position were recorded, and their average values were treated as the baseline values. If the baseline SBP was of > $140 \mathrm{mmHg}$, the patient was excluded from the study due to hypertension.

The CSE puncture was performed routinely at the level of L3-4 with the patient in the right lateral decubitus position. A 17G Tuohy needle was used to perform the epidural puncture with a paramedian approach. After identifying an entrance into the epidural space, a 27G Whitacre needle was inserted through the epidural needle. Once the cerebrospinal fluid was detected, a research isobaric ropivacaine dose of $0.75 \%$ was injected through the Whitacre needle. After injecting the ropivacaine, an epidural catheter was inserted into the epidural space by advancing it $3 \mathrm{~cm}$ through the Tuohy needle. The patient was immediately moved to the supine position with the left uterine displacement created by placing a wedge under the right hip.

In addition, an infusion of $1 \mathrm{ml} . \mathrm{kg}^{-1} \cdot \mathrm{h}^{-1}$ lactated Ringer's solution was administered until delivery. A rescue 5 -ml bolus of $2 \%$ lidocaine was administered through the epidural catheter 2 min before the surgery for patients that could still feel the cold sensation of ice being placed on the skin below the level of T6 and those that complained of pain and whose VAS score was of $>2$ points during the surgery. A 5$\mathrm{ml}$ IV dose of $0.15 \mathrm{mg} \cdot \mathrm{kg}^{-1} \mathrm{~S}$-ketamine with normal saline or $5 \mathrm{ml}$ normal saline solution was administered with a 5-ml syringe $1 \mathrm{~min}$ before the surgery, depending on group assignment. Hypotension was defined as SBP of $<80 \%$ of the baseline value. A rescue dose of $50 \mu \mathrm{g}$ phenylephrine was administered whenever hypotension was detected. Bradycardia was defined as HR of $<50$ beats per minute (bpm) and treated with $0.5 \mathrm{mg}$ atropine. The level of sedation was assessed using a 5-point scale ( 1 = agitated; 2 = alert; 3 = calm; 4 = drowsy; 5 = asleep $).{ }^{15}$

Immediately after delivery, $1 \mathrm{ml}$ of umbilical vein blood was collected by the obstetrician, and blood gas assessments were performed, using a blood gas analyser (iSTAT1 Analyzer MN:300-G, Abbott Point of Care Inc., USA) with an iSTAT CG7 + test cartridge. A patient-controlled intravenous analgesia (PCIA) pump (AM3300, ACE MEDICAL EQUIPMENT INC., Korea) was connected to the patient, and a 2-mL PCIA bolus was given at the end of the surgery; meanwhile, the patient was taught how to use the PCIA pump. 
The PCIA fluid consisted of $10 \mathrm{mg}$ hydromorphone and $90 \mathrm{ml}$ normal saline at the total volume of $100 \mathrm{ml}$. A 2-ml background infusion and a 2-ml patient-controlled analgesia (PCA) bolus was set to a 15-min lockout time. The patient was followed-up at bedside for the post-operative analgesic effect and recovery of lower limb mobility 24 and $48 \mathrm{~h}$ after surgery. The EPDS was administered at discharge.

In both RS and R groups, a research spinal dose of ropivacaine in a 5-ml syringe was prepared and given to the patient through the Whitacre needle by a specialist anaesthesiologist, who knew the exact administered dose. The remaining anaesthesiologists and all patients were blinded to the administered doses.

The spinal ropivacaine dose of $12 \mathrm{mg}$ was used for the first patient in both groups, based on findings from Tang et al. ${ }^{16}$ and Mei et al. ${ }^{17}$. The dose administered to each subsequent patient was determined by the response of the immediately preceding patient. Satisfactory anaesthetic effect was defined as the upper sensory level of spinal anesthesia not below T6 before surgery and the VAS score of $\leq 2$ points before delivery. Provided a satisfactory anaesthetic effect was achieved, the spinal dose of ropivacaine was considered suitable, and the next patient was 1/9-times as likely to receive a lower dose (decreased by $1.5 \mathrm{mg}$ ) or 8/9-times as likely to receive the same dose as the previous patient. When satisfactory anaesthetic effect was not achieved, the following patient received a dose that was increased by $1.5 \mathrm{mg}$. Minimum and maximum doses of spinal ropivacaine were $9 \mathrm{mg}$ and $18.5 \mathrm{mg}$, respectively. The dose assignment was prepared by a statistician, using the BCUD function in Microsoft Excel 2016; only the specialist anaesthesiologist had access to this information, ensuring the double-blind nature of this study.

The primary outcomes were satisfactory anaesthetic effect until delivery and the incidence of hypotension. Secondary outcomes included maternal hypotension-related symptoms and their management, upper sensory level, total co-load IV fluid volume, blood loss volume, level of sedation during surgery, recovery of lower limb mobility, analgesic effect 24 and $48 \mathrm{~h}$ after surgery, and EPDS scores at discharge; neonatal outcomes included induction-delivery interval, umbilical vein blood gas values (including $\mathrm{pH}, \mathrm{pO}_{2}, \mathrm{pCO}_{2}$, base excess $(\mathrm{BE}), \mathrm{SO}_{2}$, and lactate levels), neonatal weight, Apgar scores measured at $1 \mathrm{~min}$ and 5 min post-delivery, and the incidence of neonatal intensive care unit admission. Maternal demographic characteristics such as age, weight, height, gestational weeks, gravida, para, baseline SBP, baseline HR, and EPDS scores at admission were recorded. All primary and secondary outcomes were observed by anaesthesiologists blinded to patient group and dose assignments.

\section{Statistical analysis and sample size calculation}

This dose finding study was based on BCUD design and simulation studies that suggest that a sample size of 20-40 patients may provide stable estimates of the target dose for most realistic cases. ${ }^{18}$ The present study included 40 patients per group. 
The ED90 was defined as the spinal dose of ropivacaine associated with a $90 \%$ anaesthetic success rate and was estimated, using the isotonic regression method ${ }^{18}$. The corresponding $95 \% \mathrm{Cl}$ was obtained, using the bias-corrected percentile method with 2000 bootstrap replications. ${ }^{19}$ Isotonic regression and bootstrapping analyses were performed in R software version 3.4.4. (R Foundation for Statistical Computing, Vienna, Austria)

Demographic characteristics and secondary outcome estimates were reported as means \pm standard deviations, medians (interquartile range), and counts and proportions. Parametric data were analysed with the t-test; nonparametric data were analysed with the Mann-Whitney test. Proportions were compared, using the Chi-square and Fisher exact tests, as suitable. The peri-operative haemodynamic parameters were assessed by multiple t-tests and estimated with a two-stage linear step-up procedure of Benjamini, Krieger, and Yekutieli, with $Q=1 \%$. Statistical comparisons were made, using SPSS for Windows version 18.0 (SPSS Inc., Chicago, IL, USA) and GraphPad Prism 8 for windows (GraphPad Software, San Diego, California, USA). Statistical significance was defined as $p$-values of $<0.05$.

\section{Results}

The patient recruitment process is presented in Fig. 1. Data from 40 patients per group were included in the analysis. Maternal characteristics were similar between the groups and are presented in Table 1.

Table 1

Baseline characteristics of RS and R group.

\begin{tabular}{|llll|}
\hline Characteristics & $\begin{array}{l}\text { Group RS } \\
(\mathbf{n}=\mathbf{4 0})\end{array}$ & $\begin{array}{l}\text { Group R } \\
(\mathbf{n}=\mathbf{4 0})\end{array}$ & P value \\
\hline Age, years & $32.8 \pm 5.0$ & $31.6 \pm 3.3$ & 0.196 \\
\hline Weight, $\mathrm{kg}$ & $69.7 \pm 7.5$ & $71.4 \pm 8.9$ & 0.359 \\
\hline Height, cm & $162.8 \pm 4.9$ & $161.0 \pm 4.2$ & 0.080 \\
\hline Gestational weeks & $39.1 \pm 0.9$ & $38.9 \pm 1.1$ & 0.353 \\
\hline Gravida, $\mathrm{n}$ & $1(1 \text { to } 2)^{\mathrm{a}}$ & $1(1 \text { to } 1)^{\mathrm{a}}$ & 0.096 \\
\hline Para, $\mathrm{n}$ & $0(0 \text { to } 0)^{\mathrm{a}}$ & $0(0 \text { to } 0)^{\mathrm{a}}$ & 1.000 \\
\hline Baseline HR, bpm & $84.3 \pm 13.1$ & $89.9 \pm 14.4$ & 0.069 \\
\hline Baseline SBP, mmHg & $120.1 \pm 11.6$ & $124.0 \pm 9.8$ & 0.104 \\
\hline EPDS scores at admission & $2.9 \pm 2.3$ & $3.5 \pm 2.5$ & 0.291 \\
\hline $\begin{array}{l}\text { Values are mean } \pm \text { SD or }{ }^{\mathrm{a}} \text { median(IQR). IQR, interquartile range. HR, heart rate; SBP, systolic blood } \\
\text { pressure; EPDS, Edinburgh Postnatal Depression Scale. }\end{array}$ & \\
\hline
\end{tabular}


Figure 2 and Fig. 3 present the sequence of effective and ineffective responses to spinal ropivacaine dose in consecutive patients in the RS and R groups, respectively. The observed and pooled-adjacent-violators algorithm-adjusted response rates associated with each spinal ropivacaine dose in the RS and R groups are presented in Table 2 and Table 3, respectively. The ED90 value of spinal ropivacaine with IV Sketamine was $11.8 \mathrm{mg}$ ( $95 \% \mathrm{Cl}: 11.7$ to 12.7 ) and the corresponding value without IV S-ketamine was $14.7 \mathrm{mg}$ (95\% Cl: 14.6 to 16.0$)$. The estimated median drug saving percentage for spinal ropivacaine between the groups was $19.7 \%$ (95\% Cl: 19.0 to $26.2 \%)$.

Table 2

Observed and PAVA-Adjusted Response in RS group, showing the proportion of successful responses for each dose of spinal ropivacaine with intravenous S-ketamine. PAVA-adjusted response rates were estimated using the weighted isotonic regression method.

\begin{tabular}{|lllll|}
\hline $\begin{array}{l}\text { Assigned Dose } \\
(\mathbf{m g})\end{array}$ & $\begin{array}{l}\text { Number of } \\
\text { Successes }\end{array}$ & $\begin{array}{l}\text { Number of } \\
\text { Patients }\end{array}$ & $\begin{array}{l}\text { Observed } \\
\text { Response } \\
\text { Rate (\%) }\end{array}$ & $\begin{array}{l}\text { PAVA-adjusted Response } \\
\text { Rate(\%) }\end{array}$ \\
\hline 10.5 & 1 & 3 & 0.333 & 0.333 \\
\hline 12 & 28 & 29 & 0.966 & 0.966 \\
\hline 13.5 & 8 & 8 & 1.000 & 1.000 \\
\hline
\end{tabular}

PAVA: pooled-adjacent-violators algorithm.

Table 3

Observed and PAVA-Adjusted Response in R group, showing the proportion of successful responses for each dose of spinal ropivacaine. PAVA-adjusted response rates were estimated using the weighted isotonic regression method.

\begin{tabular}{|lllll|}
\hline $\begin{array}{l}\text { Assigned Dose } \\
(\mathbf{m g})\end{array}$ & $\begin{array}{l}\text { Number of } \\
\text { Successes }\end{array}$ & $\begin{array}{l}\text { Number of } \\
\text { Patients }\end{array}$ & $\begin{array}{l}\text { Observed } \\
\text { Response } \\
\text { Rate (\%) }\end{array}$ & $\begin{array}{l}\text { PAVA-adjusted Response } \\
\text { Rate(\%) }\end{array}$ \\
\hline 12 & 0 & 1 & 0.000 & 0.000 \\
\hline 13.5 & 4 & 7 & 0.571 & 0.571 \\
\hline 15 & 25 & 26 & 0.962 & 0.962 \\
\hline 16.5 & 6 & 6 & 1.000 & 1.000 \\
\hline
\end{tabular}

PAVA: pooled-adjacent-violators algorithm.

Maternal outcomes are presented in Table 4. The incidence of hypotension was lower in the RS group than in the $\mathrm{R}$ group $(5 \%$ vs. $65 \%, \mathrm{P}<0.001)$. The dose of rescue phenylephrine bolus administered $(0 \mathrm{mg}$ 
[0 to 0 ] vs. $50 \mathrm{mg}$ [0 to 50], $\mathrm{p}<0.001)$, and the rates of bradycardia ( $0 \%$ vs. $15 \%, \mathrm{P}=0.026)$, dyspnoea ( $0 \%$ vs. $42.5 \%, P<0.001)$, nausea ( $0 \%$ vs. $37.5 \%, P<0.001)$, and vomiting ( $0 \%$ vs. $35 \%, P<0.001)$ were lower in the RS group than in the R group. The incidence of symptoms associated with S-ketamine such as visceral traction pain $(7.5 \%$ vs. $45 \%, \mathrm{P}<0.001)$ was lower in the RS group than in the R group; in contrast, the rates of dreaming $(62.5 \%$ vs. $0 \%, \mathrm{P}<0.001)$ and dizziness $(52.5 \%$ vs. $2.5 \%, \mathrm{P}<0.001)$ were higher in the RS group than in the R group. The upper sensory level of spinal anesthesia was lower in the RS group than in the R group (T5 [4 to 5.75] vs. T4 [3 to 4], $P<0.001$ ). The levels of sedation during delivery and 5 min after delivery were significantly different between two groups $(P<0.001, P=0.001$, respectively). 
Table 4

Comparison of maternal peri-operative and post-operative outcomes between RS and R group.

\section{Outcomes}

Group RS

$(n=40)$
Group R

$(n=40)$

Peri-operative outcomes

Hypotension, \%

2(5)

26(65)

$<0.001$

Rescue phenylephrine bolus dose

$0(0 \text { to } 0)^{\mathrm{a}}$

$50(0 \text { to } 50)^{\mathrm{a}}$

$<0.001$

Hyoxemia,\%

1(2.5)

$4(10)$

0.359

Bradycardia, \%

$0(0)$

6(15)

0.026

Dyspnoea, \%

$0(0)$

$17(42.5)$

$<0.001$

Nausea, \%

$0(0)$

15(37.5)

$<0.001$

Vomiting, \%

$0(0)$

14(35)

$<0.001$

Shiver, \%

$0(0)$

$0(0)$

1.000

Visceral traction pain,\%

$3(7.5)$

Dreaming,\%

25(62.5)

18(45)

$<0.001$

Blurred vision, $\%$

$0(0)$

$0(0)$

$<0.001$

Dizziness, \%

21(52.5)

T5(4 to 5.75$)^{\mathrm{a}}$

$0(0)$

1.000

Upper sensory level

Total co-load IV fluid volume, $\mathrm{ml}$

Blood loss volume, $\mathrm{mL}$

Level of sedation before delivery

$830 \pm 194$

$1(2.5)$

$<0.001$

(score:1/2/3/4/5)

Level of sedation 5 min after delivery (score:1/2/3/4/5)

$0 / 0 / 32 / 8 / 0$

$3 / 0 / 37 / 0 / 0$

0.001

Level of sedation at the end of surgery (score:1/2/3/4/5)

$0 / 0 / 40 / 0 / 0$

$0 / 2 / 38 / 0 / 0$

0.494

Surgery time, min

$40.6 \pm 9.2$

$42.2 \pm 8.4$

0.422

Post-operative outcomes

Duration of first PCA, min

Recovery of lower limb mobility, min

$484.3 \pm 325.6$

$212.2 \pm 61.4<0.001$

$119.3 \pm 31.7$

$152.1 \pm 40.0$

$<0.001$

Values are number (proportion), mean $\pm S D$, actual numbers or amedian(IQR). IV, intravenous; PCA, patient controlled analgesia; EPDS, Edinburgh Postnatal Depression Scale; IQR, interquartile range. 


\begin{tabular}{|c|c|c|c|}
\hline Outcomes & $\begin{array}{l}\text { Group RS } \\
(n=40)\end{array}$ & $\begin{array}{l}\text { Group R } \\
(n=40)\end{array}$ & $P$ value \\
\hline Resting VAS at $24 \mathrm{H}$ & $1.4 \pm 1.1$ & $3.4 \pm 0.9$ & $<0.001$ \\
\hline Mobile VAS at $24 \mathrm{H}$ & $3.8 \pm 1.3$ & $5.9 \pm 1.3$ & $<0.001$ \\
\hline PCA times during $24 \mathrm{H}$ & $1.7 \pm 1.8$ & $2.2 \pm 2.3$ & 0.005 \\
\hline Resting VAS at $48 \mathrm{H}$ & $0.7 \pm 0.8$ & $1.8 \pm 0.7$ & $<0.001$ \\
\hline Mobile VAS at $48 \mathrm{H}$ & $2.2 \pm 1.1$ & $3.4 \pm 1.1$ & $<0.001$ \\
\hline PCA times during $24-48 \mathrm{H}$ & $0.4 \pm 0.9$ & $0.7 \pm 0.8$ & 0.123 \\
\hline EPDS scores at discharge & $2.3 \pm 2.5$ & $4.1 \pm 3.0$ & 0.004 \\
\hline
\end{tabular}

Moreover, the duration of the first PCA was longer in the RS than in the R group ( $212.2 \pm 61.4$ vs. $484.3 \pm$ $325.6 \mathrm{~min}, \mathrm{P}<0.001)$. The recovery of lower limb mobility required less time in the $\mathrm{RS}$ than in the $\mathrm{R}$ group (119.3 \pm 31.7 vs. $152.1 \pm 40.0 \mathrm{~min}, \mathrm{P}<0.001)$. Resting and mobile VAS scores at $24 \mathrm{~h}(1.4 \pm 1.1 \mathrm{vs} .3 .4 \pm$ 0.9 points, $P<0.001$ and $3.8 \pm 1.3$ vs. $5.9 \pm 1.3$ points, $P<0.001$, respectively) and $48 \mathrm{~h}(0.7 \pm 0.8$ vs. $1.8 \pm$ 0.7 points, $P<0.001$ and $2.2 \pm 1.1$ vs. $3.4 \pm 1.1$ points, $P<0.001$, respectively) were lower in the RS group than in the R group. The PCA time during $24 \mathrm{~h}$ was lower in the RS group than in the R group (1.7 $\pm 1.8 \mathrm{vs}$. $2.2 \pm 2.3$ times, $P=0.005)$. The EPDS scores at discharge were lower in the RS than in the R group $(2.3 \pm$ 2.5 vs. $4.1 \pm 3.0$ points, $P=0.004)$.

Neonatal outcomes are presented in Table 5. The pH value ( $7.37 \pm 0.03$ vs. $7.35 \pm 0.05, P=0.047)$ was different between the groups. The levels of $\mathrm{PO}_{2}(30.9 \pm 5.1 \mathrm{mmHg}$ vs. $25.2 \pm 6.2 \mathrm{mmHg}, \mathrm{P}<0.001), \mathrm{BE}$ $\left(-2.0 \pm 1.5\right.$ vs $-4.0 \pm 1.3$ mEq. $\left.\mathrm{I}^{-1}, \mathrm{P}<0.001\right)$, and $\mathrm{SO}_{2}(57.8 \pm 11.2 \%$ vs. $43.1 \pm 16.2 \%, \mathrm{P}<0.001)$ were higher, and those of lactate $\left(1.5 \pm 0.3\right.$ vs. $\left.1.8 \pm 0.4 \mathrm{mmol}^{-\mathrm{I}^{-1}}, \mathrm{P}=0.003\right)$ were lower in the $\mathrm{RS}$ group than in the $\mathrm{R}$ group. 
Table 5

Comparison of neonatal outcomes between RS and R group.

\begin{tabular}{|llll|}
\hline Outcomes & $\begin{array}{l}\text { Group RS } \\
(\mathbf{n}=\mathbf{4 0})\end{array}$ & $\begin{array}{l}\text { Group R } \\
(\mathbf{n}=\mathbf{4 0})\end{array}$ & P value \\
\hline Induction-delivery interval, min & $13.9 \pm 3.5$ & $14.9 \pm 3.7$ & 0.190 \\
\hline UV analysis & & & \\
\hline $\mathrm{pH}$ & $7.37 \pm 0.03$ & $7.35 \pm 0.05$ & 0.047 \\
\hline $\mathrm{PO} 2, \mathrm{mmHg}$ & $30.9 \pm 5.1$ & $25.2 \pm 6.2$ & $<0.001$ \\
\hline PCO2, mmHg & $40.0 \pm 4.1$ & $39.9 \pm 7.1$ & 0.931 \\
\hline BE, mEq/L & $-2.0 \pm 1.5$ & $-4.0 \pm 1.3$ & $<0.001$ \\
\hline SO2, \% & $57.8 \pm 11.2$ & $43.1 \pm 16.2$ & $<0.001$ \\
\hline Lactate, mmol/L & $1.5 \pm 0.3$ & $1.8 \pm 0.4$ & 0.003 \\
\hline Neonatal Weight, g & $3477 \pm 404$ & $3448 \pm 396$ & 0.747 \\
\hline Apgar Scores at 1 min & $10(10 \text { to } 10)^{\mathrm{a}}$ & $10(10 \text { to } 10)^{\mathrm{a}}$ & 1.000 \\
\hline Apgar Scores at 5 min & $10(10 \text { to } 10)^{\mathrm{a}}$ & $10(10 \text { to } 10)^{\mathrm{a}}$ & 1.000 \\
\hline NICU admission & $2(5)$ & $5(12.5)$ & 0.432 \\
\hline Values are number(proportion), mean $\pm \mathrm{SD}$ or ${ }^{\mathrm{a}}$ median(IQR). UV, umbilical vein. \\
\hline
\end{tabular}

Serial changes of SBP and HR values over time after spinal anesthesia administration are presented in Fig. 4. The SBP values at $4,6,8$, and $10 \mathrm{~min}(P=0.004,<0.001,<0.001,<0.001$, respectively) were higher in the RS group than in the R group. Although HR values at 2 and 4 min $(P=0.035$ and 0.028 , respectively) were higher in the $\mathrm{R}$ group than in the RS group, two-stage linear step-up procedures revealed no between-group difference in HR values.

\section{Discussion}

In this study, the ED90 of spinal ropivacaine administered with S-ketamine was $11.8 \mathrm{mg}$ ( $95 \% \mathrm{Cl}: 11.7$ to 12.7); the corresponding value without S-ketamine was $14.7 \mathrm{mg} \mathrm{(95 \%} \mathrm{Cl:} 14.6$ to 16.0$)$, derived with the BCUD method and isotonic regression. The present findings suggest that a single IV S-ketamine bolus of 0.15 mg. $\mathrm{kg}^{-1}$ may help reduce spinal ropivacaine consumption by $19.7 \%$ (95\% Cl: 19.0 to $26.2 \%$ ), helping reduce the incidence of hypotension and hypotension-related adverse effects after spinal anesthesia.

Several methods have been proposed to reduce the incidence or severity of maternal hypotension after spinal anesthesia during cesarean delivery, including the use intrathecal adjuvant opioids to decrease the 
total dose of anaesthetics used, ${ }^{8}$ prophylactic use of vasopressors, ${ }^{4,9}$ and IV pre-load colloid liquid administration. ${ }^{10}$ Zieleskiewicz et al. ${ }^{1}$ and Yao et al. ${ }^{3}$ used B ultrasound, Xu et al. ${ }^{20}$ and Toyama et al. used $^{21}$ perfusion index of peripheral vessels to predict the risk of hypotension to enable early and individualised interventions. We believe that the fundamental approach to reducing the incidence of postspinal anesthesia hypotension is to control the upper anesthesia level before delivery, while lowering intrathecal drug use and ensuring satisfactory anaesthetic effect. The present findings suggest that an ultra-low single IV bolus dose of S-ketamine may meet these criteria.

S-ketamine, the pure dextrorotatory enantiomer of ketamine, is used in clinical analgesia and anesthesia. The main effects of S-ketamine are mediated by non-competitive inhibition of the N-methyl-D-aspartate receptor; the compound also interacts with opioid receptors, monoamine receptors, and adenosine receptors. ${ }^{22}$ It is associated with dissociation (ability to communicate with the patient is retained to a certain degree), quick onset, absence of breathing function depression, and circulatory stability in shock patients, making it popular among emergency and intensive care medicine practitioners. ${ }^{22}$ In addition, it is relatively safe for infants and children, ${ }^{13}$ making S-ketamine a suitable candidate for an adjuvant IV drug during cesarean delivery with spinal anesthesia. The present study findings suggest that an ultra-low single IV bolus dose of S-ketamine may induce sleep, decrease visceral traction pain from surgical interventions, and eliminate tension in patients that undergo cesarean delivery without increasing the risk of adverse events.

Furthermore, it has been demonstrated that a low infusion rate of S-ketamine may reduce opioid consumption and the extent of postpartum delirium. ${ }^{23,24}$ In this study, the total dose of S-ketamine was even lower than that previously examined, ${ }^{23,24}$ while the post-operative analgesic effect of S-ketamine and its interactions with opioids were maintained; no case of postpartum delirium or agitation was observed in the first $48 \mathrm{~h}$. However, the rates of dreaming and dizziness were higher among the patients that received S-ketamine than among those who did not. Most dreams were associated with high-speed movements; patients experienced the feeling of being shuttled in space. Despite short duration of these side effects and their perceived acceptability among the affected patients, we recommend that patients offered this treatment are carefully briefed ahead of surgery, so they can make an informed decision.

The faster recovery of maternal lower limb mobility in the S-ketamine group was an unexpected finding, likely indicating the contribution of the lower spinal dose of ropivacaine. Satisfactory analgesic effect and faster recovery of lower limb mobility may be direct or indirect results of the ultra-low dose of Sketamine, corresponding to enhanced recovery after surgery.

Anti-depressive effects of ketamine and S-ketamine have been reported in both human and animal studies. ${ }^{25-27}$ However, studies on the impact of S-ketamine on postpartum depression are rare. Xu et al. ${ }^{28}$ demonstrated that an ultra-low single bolus dose of ketamine does not prevent postpartum depression. Brexanolone infusion is currently the standard treatment for post-partum depression. ${ }^{29}$ In this study, an ultra-low single IV bolus of S-ketamine was used after spinal anesthesia; the EPDS was administered at 
admission and discharge. The EPDS scores were similar in both groups at admission; however, at discharge, these scores were lower in the patients that did than in those that did not receive S-ketamine, suggesting S-ketamine's anti-depressive effects in the postpartum population. However, satisfactory postoperative analgesic effect may have contributed to the lower EDPS scores; further studies are required to validate the anti-depressive effect of S-ketamine in this population.

In the present study, the bolus dose of S-ketamine was $0.15 \mathrm{mg} \cdot \mathrm{kg}^{-1}$. Sen et al. ${ }^{30}$ and Menktit et al. ${ }^{31}$ previously reported that IV ketamine dose of $0.15 \mathrm{mg} \cdot \mathrm{kg}^{-1}$ may produce satisfactory analgesia, while reducing the consumption of post-operative analgesics. Given the 2:1 potency ratio of ketamine and Sketamine. ${ }^{11}$ the dose of S-ketamine should be approximately $0.075 \mathrm{mg} \cdot \mathrm{kg}^{-1}$. We tested this lower dose of S-ketamine in preliminary experiments, failing to induce sleep in most patients; consequently, the dose of $0.15 \mathrm{mg} \cdot \mathrm{kg}^{-1}$ was used, with only one patient failing to achieve sleep; this patient was sedated in the present study. Moreover, patients undergoing elective cesarean delivery without gastroesophageal reflux or full stomach were included in this study; thus, neither reflux nor aspiration were observed in any of the study patients. Although the coughing, gag, and swallowing reflexes remained functional after the administration of S-ketamine, ${ }^{22}$ the use of S-ketamine in patients with full stomach or gastroesophageal reflux disease is unsuitable. Finally, the first contact between mother and neonate is important, promoting parent-child bonding and mother's willingness to breast-feed. Although some patients remained drowsy 5 min after delivery, all patients with IV S-ketamine in the present study could be awakened at that time; all patients communicated with the neonates and were clear-headed at the end of surgery.

This study has some limitations. First, in this study, the patients were blinded to group assignment, which was known to the participating anaesthesiologists that could observe the hypnotic effect of S-ketamine. However, the primary outcomes included the optimal spinal dose of ropivacaine and incidence of hypotension after spinal anesthesia in both groups. The study was thus double-blinded. The incidence of hypotension could be observed objectively. Second, the anti-depressive effect of S-ketamine postpartum was evaluated by the EPDS after post-operative days $4-5$, by which point most patients had not recovered either physically or mentally from the impact of cesarean section. Future studies should assess the degree of postpartum depression after $\geq 6$ weeks in this population to comprehensively evaluate the anti-depressive effects of S-ketamine postpartum. Third, the low incidence of maternal hypotension and its related side effects was likely due to the decreased dose of spinal ropivacaine, associated with the use of S-ketamine. Although the effect of stimulating the sympathetic nervous system by S-ketamine has been shown in several previous studies, herein, we could not distinguish between the effect of spinal ropivacaine dose reduction and that of S-ketamine use. Establishing whether an ultra-low bolus dose of S-ketamine increases maternal BP and HR after spinal anesthesia requires further research. Finally, the study included limited parturients, thus the safety and side effects of IV S-ketamine for parturients underwent caesarean delivery need further large sample observation.

\section{Conclusion}


The ED90 of spinal ropivacaine was $11.8 \mathrm{mg}$ ( $95 \% \mathrm{Cl}: 11.7$ to 12.7 ) with and $14.7 \mathrm{mg}$ ( $95 \% \mathrm{Cl} 14.6$ to 16.0) without S-ketamine. An ultra-low single IV bolus dose of S-ketamine may reduce the required dose of spinal ropivacaine by $19.7 \%$ (95\% Cl: 19.0 to $26.2 \%)$, helping reduce the incidence of hypotension and associated symptoms, and providing sedative status during cesarean delivery. Thus a spinal ropivacaine of $12 \mathrm{mg}$ with IV S-ketamine bolus of $0.15 \mathrm{mg} \cdot \mathrm{kg}^{-1}$ could be cautiously used for parturients with high risks of post anesthesia hypotension or extreme nervousness.

\section{List Of Abbreviations}

VAS

visual analogue scale; ED90:90\% effective dose; 95\% Cl:95\% confidence interval; IV:intravenous; BCUD:biased coin up-down; ASA:American society of anesthesiologists; CSE:combined spinal and epidural; EPDS:Edinburgh Postnatal Depression Scale; SBP:Systolic blood pressure; HR:heart rate; bpm:beats per minute; UV:umbilical vein; PCIA:patient controlled intravenous analgesia; PCA:patient controlled analgesia; PAVA:pooled-adjacent-violators algorithm; NICU:neonatal intensive care unit; IQR:interquartile range;

\section{Declarations}

\section{Ethical approval and consent to participate:}

The trial was conducted in accordance with the Declaration of Helsinki (as revised in 2013). The study was approved by China Ethics Committee of Registering Clinical Trials, West Hospital, Sichuan University, Sichuan, China on 29 September 2020 (Chairperson Taixiang Wu, Ethical No.: ChiECRCT20200301) and all patients signed consent to participate before allocated to the study. The authors are accountable for all aspects of the work in ensuring that questions related to the accuracy or integrity of any part of the work are appropriately investigated and resolved.

\section{Consent for publication:}

Not applicable.

\section{Availability of data and materials:}

All data generated or analysed during this study are included in this published article [and its supplementary information files].

\section{Competing interests}

The authors declare no conflict of interest.

\section{Funding:}


Chinese Academy of Medical Sciences Research Unit (No. 2019RU056), Shanghai Jiao Tong University; CAMS Innovation Fund for Medical Sciences (CIFMS) (No. 2019-I2M-5-064). The role of both fundings are in editing the manuscript and article-processing charge.

\section{Authors' contributions}

TX, ZX designed the project; XYZ, JW, XHA, TX, YCZ collected the data; YB, TX analyzed and interpreted the data; $X Y Z$ and JW wrote the manuscript; TX and $Z X$ critical revised the manuscript; All authors reviewed and approved the final manuscript.

\section{Acknowledgements}

Not applicable.

\section{References}

1. Zieleskiewicz L, Noel A, Duclos G, et al. Can point-of-care ultrasound predict spinal hypotension during cesarean section? A prospective observational study. Anesthesia 2018;73(1):15-22. doi: 10.1111/anae.14063.

2. Orbach-Zinger S, Ginosar Y, Elliston J, et al. Influence of preoperative anxiety on hypotension after spinal anesthesia in women undergoing Cesarean delivery. Br J Anaesth 2012;109(6):943-9. doi: 10.1093/bja/aes313.

3. Yao SF, Zhao YH, Zheng J, et al. The transverse diameter of right common femoral vein by ultrasound in the supine position for predicting post-spinal hypotension during cesarean delivery. BMC Anesthesiol 2021,20;21(1):22. doi: 10.1186/s12871-021-01242-8.

4. Xu T, Zheng J, An XH, et al. Norepinephrine intravenous prophylactic bolus versus rescue bolus to prevent and treat maternal hypotension after combined spinal and epidural anesthesia during cesarean delivery: a sequential dose-finding study. Ann Transl Med 2019;7(18):451. doi: 10.21037/atm.2019.08.50

5. Kinsella SM, Carvalho B, Dyer RA, et al. International consensus statement on the management of hypotension with vasopressors during cesarean section under spinal anesthesia. Anesthesia 2018;73(1):71-92. doi: 10.1111/anae.14080.

6. Onwochei DN, Ngan Kee WD, Fung L, et al. Norepinephrine intermittent intravenous boluses to prevent hypotension during spinal anesthesia for cesarean delivery: a sequential allocation dosefinding study. Anesth Analg 2017;125:212-8. doi: 10.1213/ANE.0000000000001846.

7. Vallejo MC, Attaallah AF, Elzamzamy OM, et al. An open-label randomized controlled clinical trial for comparison of continuous phenylephrine versus norepinephrine infusion in prevention of spinal hypotension during cesarean delivery. Int J Obstet Anesth 2017; 29:18-25. doi: 10.1016/j.ijoa.2016.08.005. 
8. Chen X, Qian X, Fu F, et al. Intrathecal sufentanil decreases the median effective dose (ED50) of intrathecal hyperbaric ropivacaine for cesarean delivery. Acta Anaesthesiol Scand 2010; 54(3):28490. doi: 10.1111/j.1399-6576.2009.02051.x.

9. Zheng J, An X, Qian JY, et al. Research letter: ED(90) of phenylephrine prophylactic bolus dose to prevent maternal hypotension during cesarean delivery. J Clin Anesth 2020, 16;64:109812. doi: 10.1016/j.jclinane.2020.109812.

10. Loubert C, Gagnon PO, Fernando R. Minimum effective fluid volume of colloid to prevent hypotension during cesarean section under spinal anesthesia using a prophylactic phenylephrine infusion: An updown sequential allocation study. J Clin Anesth 2017;36:194-200. doi:

10.1016/j.jclinane.2016.10.018.

11. Weber F, Wulf H, el Saeidi G. Premedication with nasal s-ketamine and midazolam provides good conditions for induction of anesthesia in preschool children. Can J Anaesth 2003;50(5):470-5. doi: 10.1007/BF03021058.

12. Timm C, Linstedt U, Weiss $T$, et al. Sympathomimetic effects of low-dose S(+)-ketamine. Effect of propofol dosage. Anaesthesist 2008;57(4):338-46. doi: 10.1007/s00101-008-1331-0.

13. Pees $C$, Haas NA, Ewert $P$, et al. Comparison of analgesic/sedative effect of racemic ketamine and $\mathrm{S}(+)$-ketamine during cardiac catheterization in newborns and children. Pediatr Cardiol 2003;24(5):424-9. doi: 10.1007/s00246-002-0356-4.

14. Smith-Nielsen J, Matthey S, Lange T, et al. Validation of the Edinburgh Postnatal Depression Scale against both DSM-5 and ICD-10 diagnostic criteria for depression. BMC Psychiatry 2018, 20;18(1):393. doi: 10.1186/s12888-018-1965-7.

15. Bhat R, Santhosh MC, Annigeri VM, et al. Comparison of intranasal dexmedetomidine and dexmedetomidine-ketamine for premedication in pediatrics patients: A randomized double-blind study. Anesth Essay Res 2016;10(2):349-55. doi: 10.4103/0259-1162.172340.

16. Tang Y, Yang M, Fu F, et al. Comparison of the ED50 of intrathecal hyperbaric ropivacaine coadministered with or without intrathecal dexmedetomidine for cesarean section: A prospective, double-blinded, randomized dose-response trial using up-down sequential allocation method. J Clin Anesth 2020;62:109725. doi: 10.1016/j.jclinane.2020.109725.

17. Mei Z, Ngan Kee WD, Sheng ZM, et al. Comparative dose-response study of hyperbaric ropivacaine for spinal anesthesia for cesarean delivery in singleton versus twin pregnancies. J Clin Anesth 2020;67:110068. doi: 10.1016/j.jclinane.2020.110068.

18. Pace NL, Stylianou MP. Advances in and limitations of up-and-down methodology: a précis of clinical use, study design, and dose estimation in anesthesia research. Anesthesiology 2007;107:144-52. doi: 10.1097/01.anes.0000267514.42592.2a.

19. Stylianou M, Proschan M, Flournoy N. Estimating the probability of toxicity at the target dose following an up and-down design. Stat Med 2003;22:535-43. doi: 10.1002/sim.1351.

20. Xu Z, Xu T, Zhao P, Ma R, Zhang M, Zheng J. Differential roles of the right and left toe perfusion index in predicting the incidence of postspinal hypotension during cesarean delivery. Anesth Analg 
2017;125(5):1560-6. doi: 10.1213/ANE.0000000000002393.

21. Toyama $S$, Kakumoto $M$, Morioka $M$, et al. Perfusion index derived from a pulse oximeter can predict the incidence of hypotension during spinal anesthesia for cesarean delivery. $\mathrm{Br} \mathrm{J}$ Anaesth 2013;111(2):235-41. doi: 10.1093/bja/aet058.

22. Trimmel $\mathrm{H}$, Helbok R, Staudinger T, et al. S(+)-ketamine Current trends in emergency and intensive care medicine. Wien Klin Wochenschr 2018;130(9-10):356-366. doi: 10.1007/s00508-017-1299-3.

23. Bornemann-Cimenti $H$, Wejbora M, Michaeli $K$, et al. The effects of minimal-dose versus low-dose sketamine on opioid consumption, hyperalgesia, and postoperative delirium: a triple-blinded, randomized, active- and placebo-controlled clinical trial. Minerva Anestesiol 2016;82(10):1069-1076.

24. Suppa E, Valente A, Catarci S, et al. A study of low-dose S-ketamine infusion as "preventive" pain treatment for cesarean section with spinal anesthesia: benefits and side effects. Minerva Anesthesiol 2012;78(7):774-81.

25. Swainson J, Thomas RK, Archer S, et al. Esketamine for treatment resistant depression. Expert Rev Neurother 2019;19(10):899-911. doi: 10.1080/14737175.2019.1640604.

26. Corriger A, Pickering G. Ketamine and depression: a narrative review. Drug Des Devel Ther 2019,27;13:3051-3067. doi: 10.2147/DDDT.S221437.

27. Yang C, Shirayama Y, Zhang JC, et al. R-ketamine: a rapid-onset and sustained antidepressant without psychotomimetic side effects. Transl Psychiatry 2015;5(9):e632. doi: 10.1038/tp.2015.136.

28. Xu Y, Li Y, Huang X, et al. Single bolus low-dose of ketamine does not prevent postpartum depression: a randomized, double-blind, placebo-controlled, prospective clinical trial. Arch Gynecol Obstet 2017;295(5):1167-1174. doi: 10.1007/s00404-017-4334-8.

29. Cristea LA, Naudet F. US Food and Drug Administration approval of esketamine and brexanolone. Lancet Psychiatry 2019;6(12):975-977. doi: 10.1016/S2215-0366(19)30292-5.

30. Sen S, Ozmert G, Aydin ON, et al. The persisting analgesic effect of low-dose intravenous ketamine after spinal anesthesia for Cesarean section. Eur J Anaesthesiol 2005;22(7):518-23. doi: $10.1017 / s 026502150500089 x$.

31. Menkiti ID, Desalu I, Kushimo OT. Low-dose intravenous ketamine improves postoperative analgesia after cesarean delivery with spinal bupivacaine in African parturients. Int J Obstet Anesth 2012;21(3):217-21. doi: 10.1016/j.ijoa.2012.04.004.

\section{Figures}




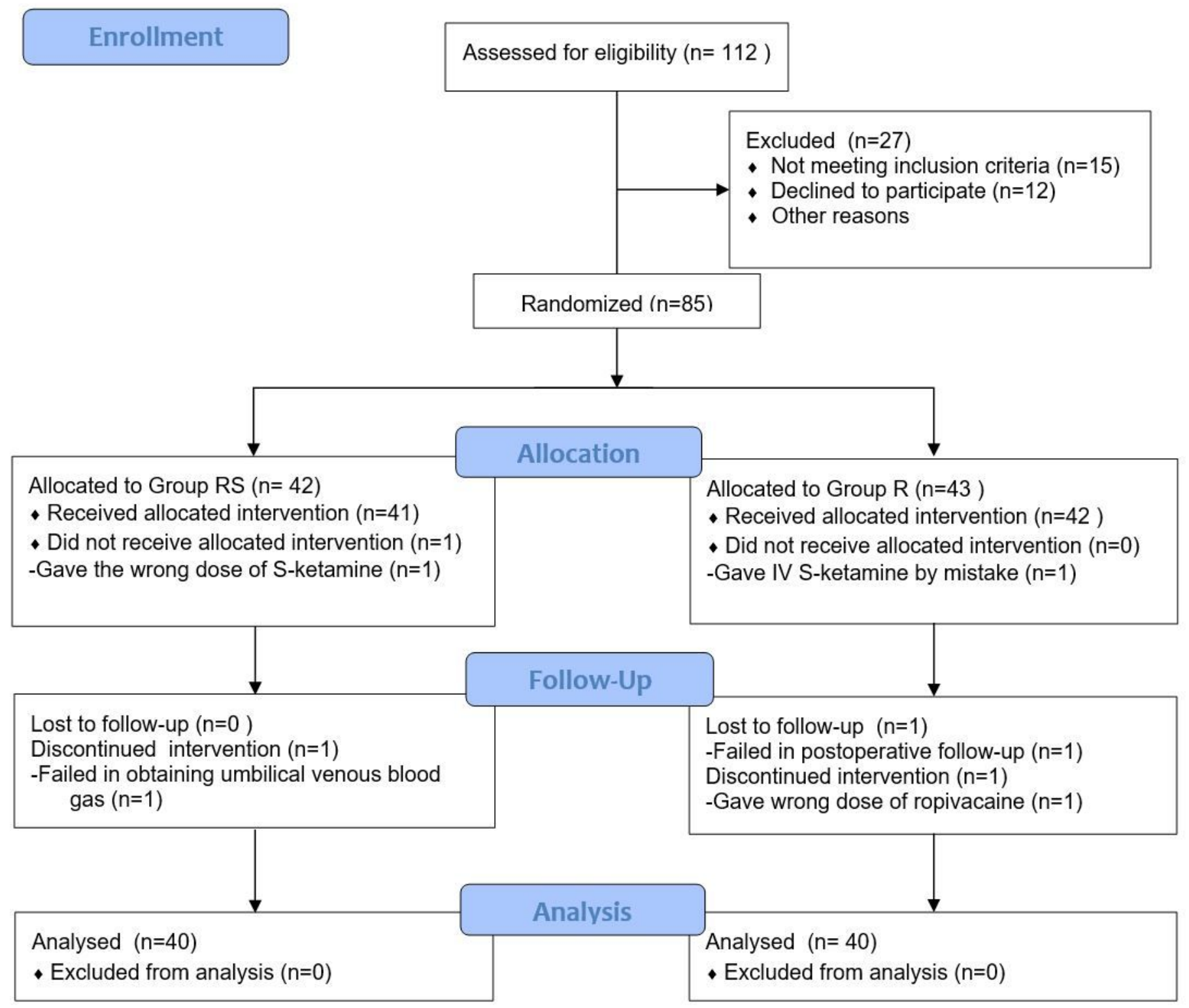

Figure 1

Flow chart of the study. 


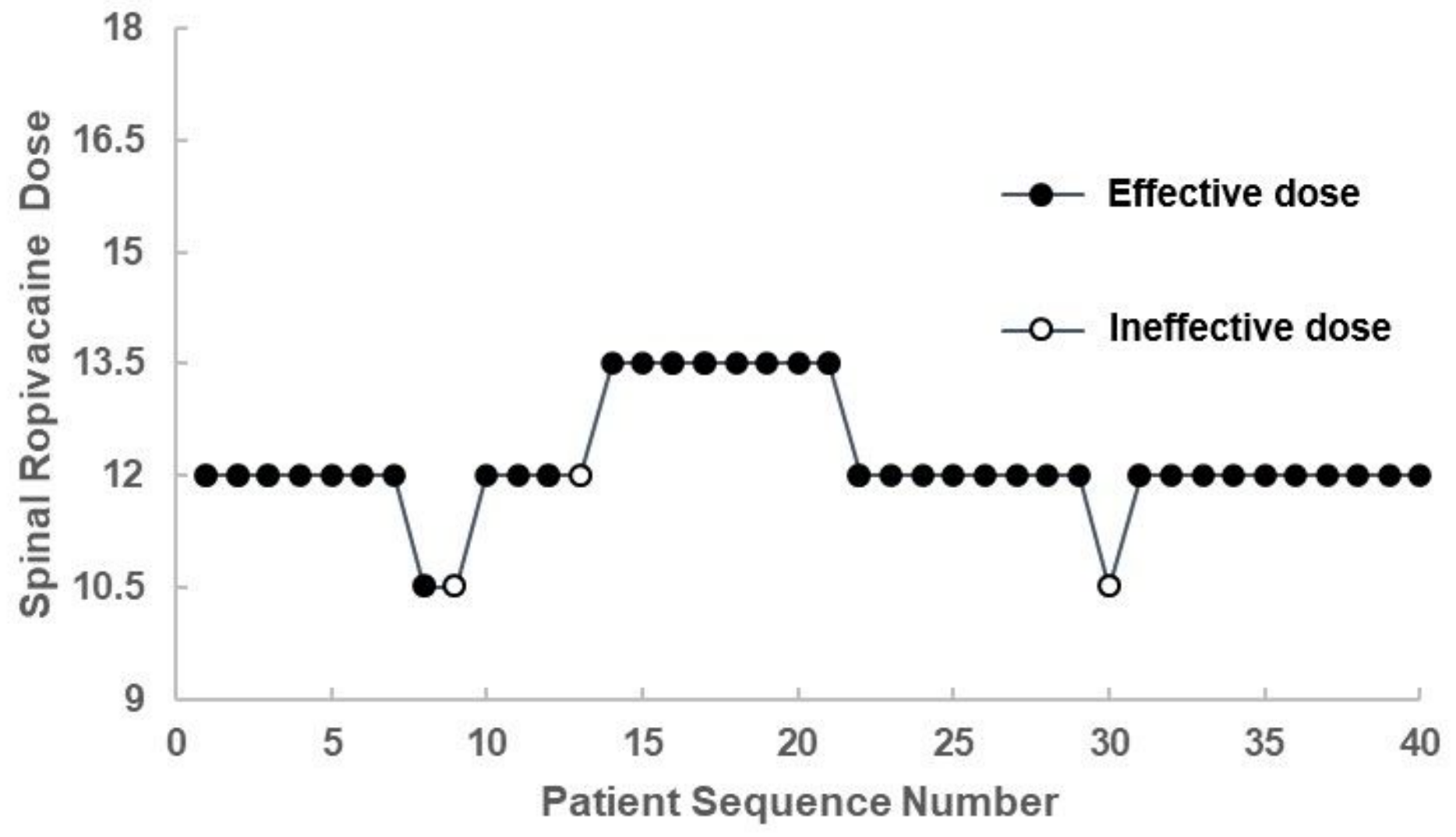

Figure 2

The patient allocation sequence and the response to the assigned dose of spinal ropivacaine in RS group. The patient sequence number ( $\mathrm{X}$-axis) is the order of patient exposures using the BCUD design. The assigned dose levels are presented on Y-axis. An effective dose is denoted by a solid circle, while an ineffective one is denoted by a hollow circle. BCUD, biased coin up and down. 


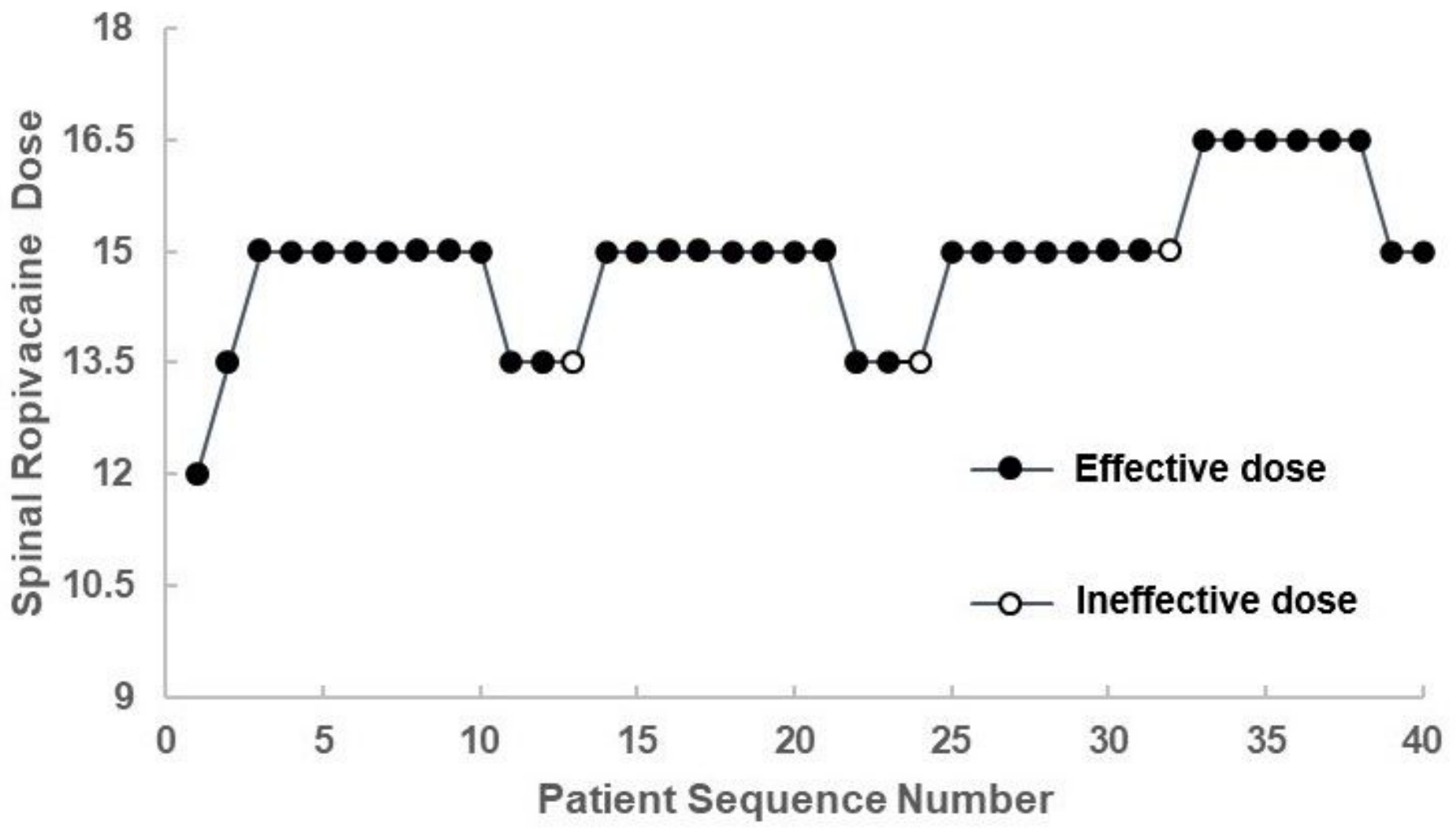

Figure 3

The patient allocation sequence and the response to the assigned dose of spinal ropivacaine in $\mathrm{R}$ group. The patient sequence number (X-axis) is the order of patient exposures using the BCUD design. The assigned dose levels are presented on Y-axis. An effective dose is denoted by a solid circle, while an ineffective one is denoted by a hollow circle. BCUD, biased coin up and down.
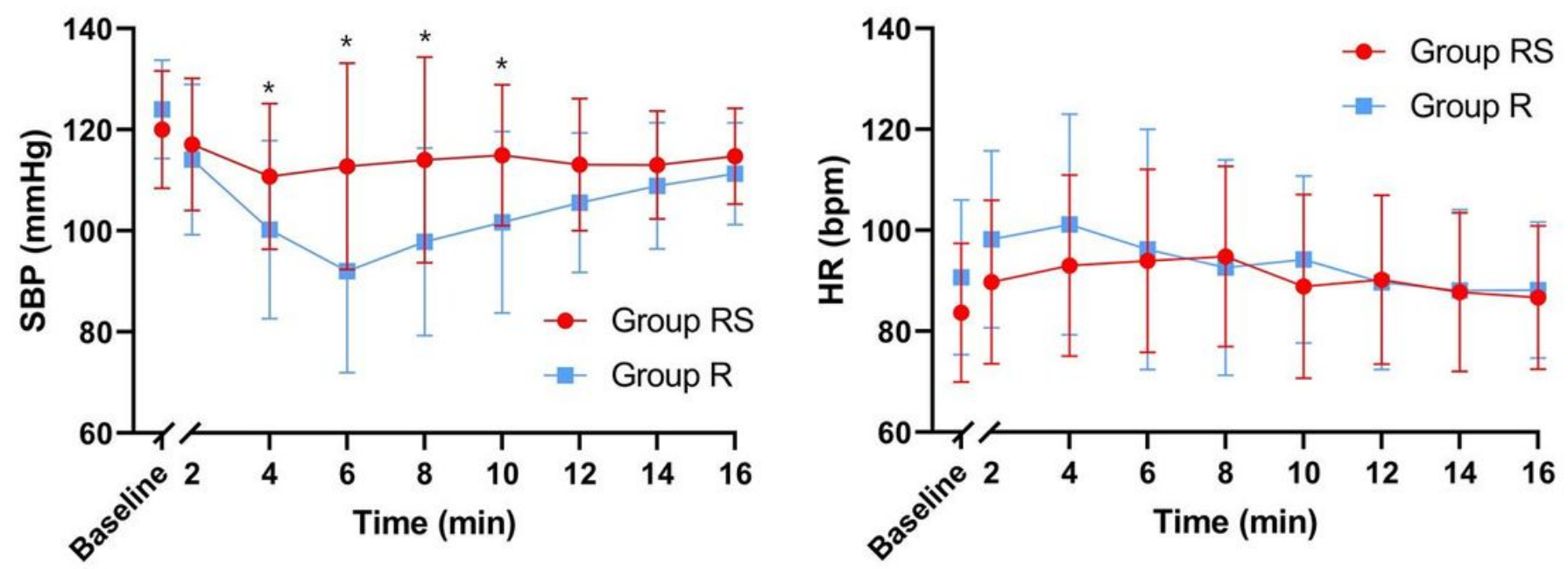


\section{Figure 4}

Serial changes of hemodynamic over time after the spinal anesthesia of two groups. The red circles and their error bars represent the means and SDs of SBP and HR over times of 40 patients in RS group; The blue squares and their error bars represent the means and SDs of SBP and HR over times of 40 patients in R group. (a) Serial changes of SBP over time. (b) Serial changes of HR over time. *Significant difference between the two groups were assessed by multiple t test and determined by Two-stage linear step-up procedure of Benjamini, Krieger and Yekutieli, with $Q=1 \%$. SBP, systolic blood pressure; HR, heart rate; bmp, beats per minute.

\section{Supplementary Files}

This is a list of supplementary files associated with this preprint. Click to download.

- CONSORT2010Checklist.doc 